\title{
Acne associated gynecological diseases and risk factors in the multiethnic women
}

\begin{abstract}
The objective: To identify, review and compare the pathological acne and the connection between other gynecological diseases in women of different ethnic groups.

Methods and materials: This study was carried out based on the World Health Organization database, hospital based acne incidence, different research materials and official websites of medical committees.

Results: After analyzing the materials for 20 years, one in ten (7-17\%) of people having acne after the age of twenty five. Women are far more likely to have acne during their early adult years than men, with more than eight in ten of cases of adult acne occurring in women. Clinical acne was more prevalence in African American (37\%) and Hispanic women (32\%) than in Continental Indian (23\%), Caucasian (24\%) and Asian women (30\%).Among the women with acne $37.3 \%$ of women were diagnosed with polycystic ovarian syndrome where as $39.2 \%$ with abnormal menstruation. Other percentage of women diagnosed with premenstrual dysphoric disorder and other gynecological disorders.

Conclusion: Many causes of adult acne are due to changes in hormone levels that women experience at certain points during their lives such as before menstrual periods, during pregnancy, starting or stopping birth control pills and polycystic ovarian syndrome and their prevalence percentage depend on their nationality. Importantly, public health initiatives that improve acne associated with gynecological diseases awareness address amenable risk factors and allow for the early detection will be essential in addressing the outcome inequalities that currently exist.
\end{abstract}

Keywords: acne, polycystic ovarian syndrome, hirsutism, Hormones, risk factors, prevention
Volume 10 Issue I - 2019

\author{
Khuraseva $A B, '$ Jayaweera JACS 2 \\ 'Doctor of Medical Science, Department of Obstetrics and \\ Gynecology, Kursk state medical university, Russian Federation, \\ Russia \\ ${ }^{2}$ Undergraduate student, Kursk state medical university, Kursk, \\ Russian Federation, Russia
}

\begin{abstract}
Correspondence: Jayaweera JACS, Undergraduate student, Kursk state medical university, Kursk, Russian Federation, Russia, Tel +79524960626, Email chira.jaya@gmail.com
\end{abstract}

Received: January 14, 2019 | Published: January 30, 2019

\section{Introduction}

Acne is the most common type of inflammatory dermatological disease widespread among any age from newborn to menopause .Peak incidence of acne (59\% to 95\%) occurs in adolescent girls aged between 15-16. After 25 years the frequency of acne decreases up to $25 \%{ }^{1,2}$ Acne is thought to be caused by changes in hormones that are triggered during puberty and frequency of occurrence doesn't depend on gender. But women of secondary reproductive age suffer more from acne than men. Psychological stress, diet, smoking, genetic predisposition and hormonal disbalance have been considered as factors that can trigger or worsen acne. Research studies have shown that there is a direct relationship of acne with hormonal disbalance and gynecological diseases. ${ }^{3}$

\section{Can stress exacerbate acne?}

There is increasing evidence that psychological stress is an important factor in acne pathogenesis. Emotional stress associated with the production of hormones, neuro peptides, and inflammatory cytokines influences the chronic course and exacerbation of acne by altering the activity of the pilosebaceous unit. These mechanisms involve the HPA axis and the neuro-immuno-cutaneous system where neuropeptides and hormones such as $\mathrm{CRH}$ and melanocortins play a substantial role. ${ }^{4}$ Stress signals initiate the hypothalamic pituitary adrenal (HPA) axis and the sympathetic nervous system, while also inducing secretion of different neurotransmitters, cytokines, and hormones that possess skin receptors and can aggravate several skin diseases, including acne. ${ }^{5,6}$ The corticotrophin-releasing hormone (CRH) appears to be an important aspect in the development of acne lesions. ${ }^{78}$ Many patients report that emotional stress makes their acne worse, and these statements were confirmed in several studies by a significant percentage of affected adolescents and adults (varying between 50-80\%). ${ }^{9}$ An Australian survey that included 215 graduating medical students, reported that $67 \%$ of them identified stress as one of the factors leading to acne exacerbations. ${ }^{10}$ Two Korean epidemiological studies found psychological stress to be the main triggering or aggravating factor influencing acne as reported by the majority $(80-82 \%)$ of patients (Figure 1$).{ }^{9}$

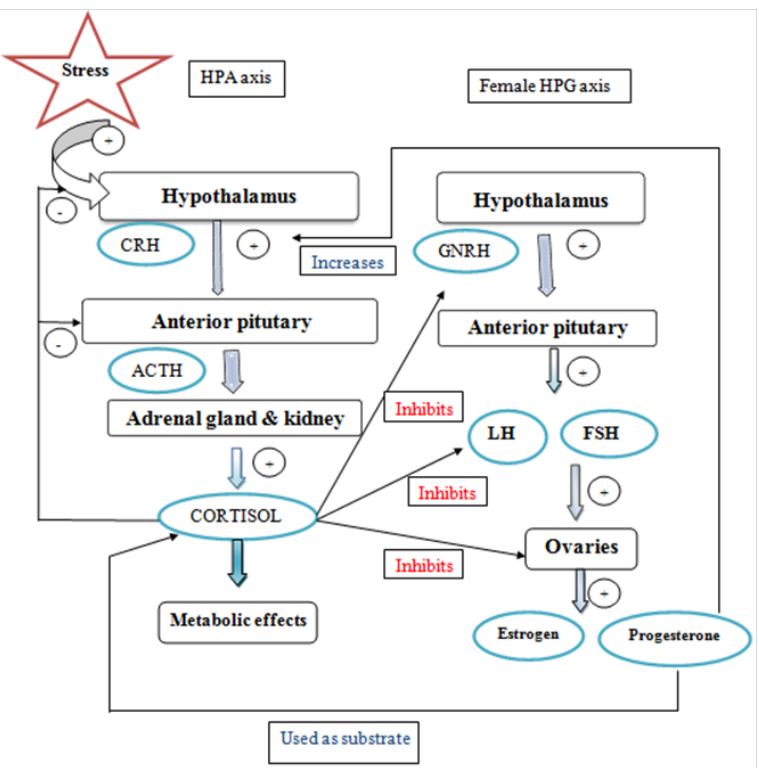

Figure I Effects of stress on acne (www.thepaleomom.com). 


\section{The effects of smoking on acne}

Smoking has negative effect on human body including acne. Some studies prove that nicotine has an anti-inflammatory action on acne. ${ }^{11}$ According to recent studies it showed that smokers have higher affinity to acne and there is a relationship between smoking and noninflammatory acne. ${ }^{12}$ Smoking produce free radicals it stimulate body to produce cytokines. ${ }^{13}$

Studies have shown that cytokine levels are high in clogged pores and when healthy skin is exposed to high levels of cytokines; it starts to develop acne. ${ }^{14}$ Smoking leads to the reduction of estrogen levels in the body and it leads to ovarian malfunction, irregular menstruation. According to the researches smoking has less effect on changing testosterone levels in the body. ${ }^{15}$

\section{Relationship between acne and hormone levels}

Hormones are an important factor in acne development, particularly androgens, which are present in both males and females. Higher levels of androgens tend to lead to acne. While it is not known exactly how hormones affect acne, we do know that the skin is a target for hormones and can even produce hormones itself. ${ }^{16}$ Higher levels of estrogens normally correlate with fewer acne symptoms (Figure 2).

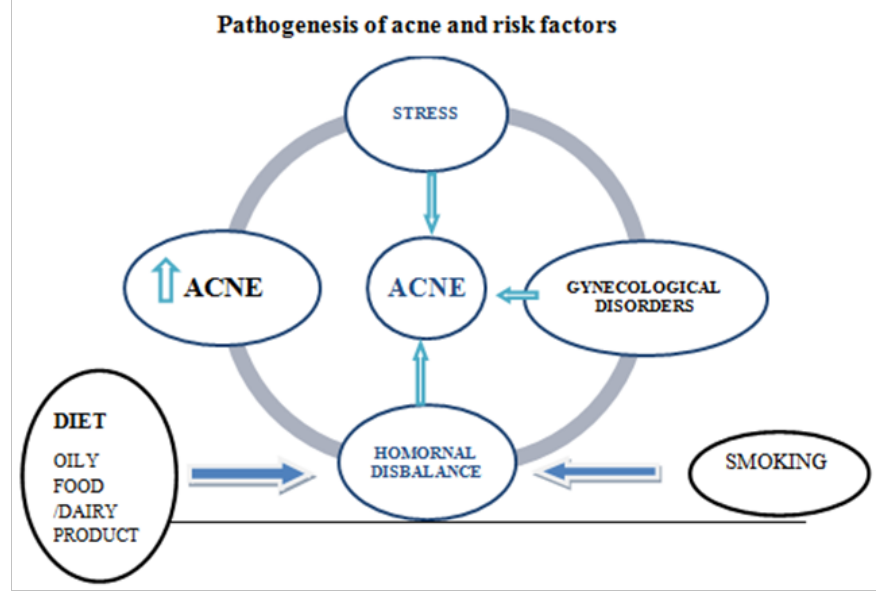

Figure 2 Jayaweera JACS, Khuraseva AB. Acne association with gynecological diseases in women of different nationality 2017.

\section{Effects of food on acne}

\section{Dairy products}

Milk contains insulin-like growth factor (IGF-1). Higher levels of IGF-1 lead to higher production of skin oil. Higher production of skin oil because more prevalence of acne, researchers states that insulinlike growth factor in milk increased skin oil production and cause higher production of acne. Milk contains androgen precursors. These precursors require enzymes to convert them into actual male hormones in the body, and these enzymes are readily available in the pores of the skin. ${ }^{17}$

\section{Chocolate}

There is a correlation between chocolate and increased insulin levels. Higher levels of insulin lead to higher incidence of acne symptoms by increasing skin cell production. It cause increased skin oil (sebum) production and clogged pores which provide suitable environment for acne bacteria. Chocolate consists of antioxidants, which might theoretically help to reduce acne symptoms. Dark chocolate leads to the lowering of blood pressure which may lead to increase oxygen and nutrient distribution to dermis and help to relieve acne associated symptoms. ${ }^{18}$

\section{Fatty acid/oil}

According to researches fatty diets lead higher fat content in sebum. Omega-3s are known to improve mood and reduce stress and anxiety. Since there is a correlation between stress and acne omega-3 fats could help in reduction of acne symptoms. ${ }^{19}$

\section{Acne-associated syndromes}

Acne-associated syndromes prove the nature of these diseases and are indicative of pathogenesis of acne. Polycystic ovary (PCOS), synovitis-acne-pustulosis-hyperostosis-osteitis (SAPHO), hyperandrogenism-insulin resistance-acanthosis nigricans (HAIRAN), pyogenic arthritis-pyoderma gangrenosum-acne (PAPA), pyoderma gangrenosum-acne vulgaris-hidradenitis suppurativaankylosing spondylitis (PASS), pyoderma gangrenosum-acne conglobate-hidradenitis suppurativa (PASH), seborrhea-acnehirsutism-androgenic alopecia (SAHA), and Apert syndromes are well known acne associated syndromes. Endocrine disorders (insulin resistance, obesity, hyperandrogenism, etc.) can be commonly seen in this syndromes. ${ }^{20}$

\section{Polycystic ovary syndrome (PCOS)}

Polycystic ovary syndrome (PCOS) is an ovarian disease characterized by hyperandrogenism, chronic anovulation, and polycystic ovaries. It is one of the most common endocrinopathy that affects $4-12 \%$ of women of reproductive age. ${ }^{21}$ The pulse frequency of gonadotropin-releasing hormone $(\mathrm{GnRH})$ increases in PCOS and stimulates to the anterior pituitary gland to secrete luteinizing hormone ( $\mathrm{LH})$ more than follicle-stimulating hormone (FSH), resulting in an increased ratio of LH to FSH. Studies have shown that disruption of production and the ratio of gonadotropic hormones (FSH and $\mathrm{LH}$ ) found in $23.9 \%$ of patients with acne. ${ }^{3}$ The increase in LH relative to FSH stimulates the ovarian theca cells to synthesize androstenedione. Consequently, the net ovarian androgen production increases. ${ }^{6}$ Insulin has also a role in the pathogenesis of PCOS by stimulating the ovarian theca cell to secrete androgens as LH and also inhibits hepatic production of sex hormone binding globulin (SHBG). As a result, free and total androgen level increases. Insulin resistance and hyperandrogenism are responsible for the cutaneous involvement of PCOS. Insulin resistance causes acanthosis nigricans (AN), and hyperandrogenism leads to hirsutism, acne, oily skin, seborrhea, and hair loss (androgenic alopecia). It is estimated that $72-82 \%$ of women with PCOS have cutaneous signs. ${ }^{22}$ PCOS has also multisystem effects and is associated with lots of diseases including infertility, endometrial cancer, obesity, depression, sleep-disordered breathing/obstructive sleep apnea (OSA), nonalcoholic fatty liver disease (NAFLD) and nonalcoholic steatohepatitis (NASH), type 2 diabetes mellitus (T2DM), and cardiovascular diseases. ${ }^{23}$ Although acne, hirsutism, and were the most common skin manifestations, hirsutism and were the most sensitive for PCOS diagnosis. According to research reports $32.8 \%$ patients with acne got PCOS. ${ }^{3}$

\section{Hyperandrogenism-insulin nigricans syndrome (HAIR-AN syndrome)}

Hyperandrogenism-insulin resistance-acanthosis nigricans syndrome (HAIR-AN syndrome) is a subphenotype of polycystic ovary syndrome. It is clinically characterized by acne, obesity, hirsutism, and acanthosis nigricans. It usually manifests in early adolescence. Genetic, environmental factors, and obesity are 
estimated to cause HAIR-AN syndrome. ${ }^{24}$ Adolescents with HAIRAN syndrome usually have normal levels of luteinizing hormone (LH) and follicle-stimulating hormone (FSH) but the ratio of LH to FHS is usually more than one. ${ }^{25}$ Polycystic ovary syndrome (71-86\%), congenital hyperplasia of the adrenal (3-10\%), adrenal and ovarian tumors $(0.3 \%)$, and idiopathic hirsutism $(10 \%)$ are, respectively, the most common reasons of hyperandrogenism. Except those, HAIR-AN syndrome is a reason of hyperandrogenism that is seen in almost $5 \%$ of females with hyperandrogenism. ${ }^{26}$

\section{SAHA syndrome (seborrhea-acne-hirsutism- androgenic alopecia)}

The SAHA syndrome is classified into four types: idiopathic, ovarian, adrenal, and hyperprolactinemic, and it can be associated with polycystic ovaries, cystic mastitis, obesity, insulin resistance, and infertility. ${ }^{27}$ In the pathogenesis of SAHA, increased androgen synthesis in adrenals and ovaries, disturbed peripheral metabolism of androgens or induction of metabolism and activation of androgens in the skin may play important role. Approximately $20 \%$ of the patients have all four major signs of SAHA syndrome. Seborrhoea is observed in all of patients, androgenetic alopecia is seen in $21 \%$ of the patients, and acne in $10 \%$ and hirsutism in $6 \%$ of the patients. ${ }^{28}$

\section{Prevalence of acne in multiethnic woman}

Asian women showed slightly lower acne incidence compared to African American or Hispanic women, but slightly higher compared to Caucasians and Indians. Asian women had the lowest amount of noninflammatory blackheads and whiteheads, than any other ethnicity but they had the highest amount of inflammatory acne. ${ }^{29}$ Black skin woman mostly has inflammatory acne nodules and cysts. ${ }^{30}$

Based on the Journal of the European Academy of Dermatology and Venereology 2011 clinical acne was more prevalent in African American and Hispanic women (37\%, 32\% respectively) than in Continental Indian, Caucasian and Asian $(23 \%, 24 \%, 30 \%$ respectively) women. All racial groups displayed equal prevalence of both subtypes of acne with the exception of Asians, for whom inflammatory acne was more prevalent than comedonal (20\% vs. $10 \%)$ acne, and in Caucasians, for whom comedonal acne was more prevalent than inflammatory ( $14 \%$ vs. $10 \%)$ acne. Hyper pigmentation was more prevalent in African American and Hispanic (65\%, 48\% respectively) than in Asian, Continental Indian and Caucasian (18\%, $10 \%$, and $25 \%$ respectively) women. ${ }^{31,32-40}$

\section{Conclusion}

Their lives before menstrual periods, during pregnancy, starting or stopping birth control pills, polycystic ovarian syndrome, hyperandrogenism-insulin resistance-acanthosis nigricans (HAIRAN), seborrhea-acne-hirsutism-androgenic alopecia (SAHA).

When considering the Acne in different ethnic groups genetic predisposing factors for androgen receptors is highest in Black African woman, lowest in White Caucasian woman and moderate in Asian woman.

Black skin is tougher than white skin and is less sensitive towards many factors, such as sunlight. Prevalence of acne in black skin is more than in white skin. White skin is generally more sensitive and it is not that rough and tough and the acne on white skin is more visible and clear than any other skin color. Acne is more prevalent in white skin due to several genetic factors and their life style modification.
Healthy food consumption and healthy life style, early screening for gynecological diseases, early treatment for hormonal disbalance, proper contraception methods will help to reduce the prevalence of acne related gynecological diseases in woman.

\section{Acknowledgments}

None.

\section{Conflicts of interest}

The authors declare no conflicts of interest.

\section{References}

1. Amado JM, Matos ME, Abreu AM, et al. The prevalence of acne in the north of Portugal. J Eur Acad Dermatol Venereol. 2006;20(10):12871295.

2. Collier CN, Harper JC, Cafardi JA, et al. The prevalence of acne in adults 20 years and older. J Am Acad Dermatol. 2008;58(1):56-59.

3. Huraseva AB. Acne: not only a cosmetic problem. Modern gynecology. 2018;2(13):31-34

4. Jović A, Marinović B, Kostović K, et al. The Impact of Pyschological Stress on Acne. Acta Dermatovenerol Croat. 2017;25(2):133-141.

5. Bowe W, Patel NB, Logan AC. Acne vulgaris, probiotics and the gutbrain-skin axis: from anecdote to translational medicine. Benef Microbes. 2014;5(2):185-199.

6. Knowles SR, Nelson EA, Palombo EA. Investigating the role of perceived stress on bacterial flora activity and salivary cortisol secretion: a possible mechanism underlying susceptibility to illness. Biol Psychol. 2008;77(2):132-137.

7. Poli F, Dreno B, Verschoore M. An epidemiological study of acne in female adults: results of a survey conducted in France. J Eur Acad Dermatol Venereol. 2001;15(6):541-545.

8. Slominski A. On the role of the corticotropin-releasing hormone signalling system in the aetiology of inflammatory skin disorders. $\mathrm{Br} J$ Dermatol. 2009;160(2):229-232.

9. Suh DH, Kim BY, Min SU, et al. A multicenter epidemiological study of acne vulgaris in Korea. Int J Dermatol. 2011;50(6):673-681.

10. Green J, Sinclair RD. Perceptions of acne vulgaris in final year medical student written examination answers. Australas J Dermatol. 2001;42(2):98-101.

11. Firooz A, Sarhangnejad R, Davoudi SM, et al. Acne and smoking: is there a relationship? BMC Dermatol. 2005;5:2.

12. Schafer T, Nienhaus A, Vieluf D, et al. Epidemiology of acne in the general population: the risk of smoking. Br J Dermatol. 2001;145(1):100-104.

13. Carnevale R, Sciarretta S, Violi F, et al. Acute impact of tobacco vs electronic cigarette smoking on oxidative stress and vascular function. Chest. 2016;150(3):606-612.

14. Szabo K, Tax G, Kis K, et al. Interleukin- $1 \mathrm{~A}+4845(\mathrm{G}>\mathrm{T})$ polymorphism is a factor predisposing to acne vulgaris. Tissue Antigens. 2010;76(5):411415 .

15. Kapoor D, Jones TH. Smoking and hormones in health and endocrine disorders. Eur J Endocrinol. 2005;152(4):491-499.

16. Chen WC, Zouboulis CC. Hormones and the pilosebaceous unit. Dermatoendocrinol. 2009;1(2);81-86.

17. Chen W, Thiboutot D, Zouboulis C. Cutaneous androgen metabolism: basic research and clinical perspectives. $J$ Invest Dermatol. 2002;119(5):992-1007. 
18. Davidovici BB, Wolf R. The role of diet in acne: facts and controversies. Clin Dermatol. 2010;28(1):12-16.

19. Bowe WP, Joshi SS, Shalita AR. Diet and acne. J Am Acad Dermatol. 2010;63(1):124-141.

20. Nazan Emiroglu. Acne-associated syndromes. 2017.

21. Norman RJ, Dewailly D, Legro RS, et al. Polycystic ovary syndrome. Lancet. 2007; 370(9588):685-697.

22. Schmidt TH, Shinkai K. Evidence-based approach to cutaneous hyperandrogenism in women. J Am Acad Dermatol. 2015; 73(4):672690.

23. Legro RS, Arslanian SA, Ehrmann DA, et al. Diagnosis and treatment of polycystic ovary syndrome: an endocrine society clinical practice guideline. J Clin Endocrinol Metab. 2013;98(12):4565-4592.

24. Dédjan AH, Chadli A, El Aziz S, et al. Hyperandrogenism-Insulin resistance-acanthosis nigricans syndrome. Case Rep Endocrinol. $2015 ; 193097$

25. Omar HA, Logsdon S, Richards J. Clinical profiles, occurrence, and management of adolescent patients with HAIR-AN syndrome. Sci World J. 2004;4:507-511

26. Escobar-Morreale HF, Carmina E, Dewailly D, et al. Epidemiology, diagnosis and management of hirsutism: a consensus statement by the androgen excess and polycystic ovary syndrome society. Hum Reprod Update. 2012;18(2):146-170.

27. Dalamaga M, Papadavid E, Basios G, et al. Ovarian SAHA syndrome is associated with a more insulin-resistant profile and represents an independent risk factor for glucose abnormalities in women with polycystic ovary syndrome: a prospective controlled study. J Am Acad Dermatol. 2013;69(6):922-930.

28. Chen W, Obermayer-Pietsch B, Hong JB, et al. Acne-associated syndromes: models for better understanding of acne pathogenesis. $J$ Eur Acad Dermatol Venereol. 2011;25(6):637-646.
29. Taylor SC, Cook-Bolden F, Rahman Z, et al. Acne vulgaris in skin of color. J Am Acad Dermatol. 2002;46 2 Suppl Understanding):S98-S106.

30. Tan HH, Tan AW, Barkham T, et al. Community-based study of acne vulgaris in adolescents in Singapore. Br J Dermatol. 2007;157(3):547551.

31. Perkins AC, Cheng CE, Hillebrand GG, et al. Comparison of the epidemiology of acne vulgaris among Caucasian, Asian, Continental Indian and African American women. J Eur Acad Dermatol Venereol. 2011;25(9):1054-1060.

32. Adebamowo CA, Spiegelman D, Danby FW, et al. High school dietary dairy intake and teenage acne. J Am Acad Dermatol. 2005;52(2):207214.

33. Arora MK, Seth S, Dayal S. The relationship of lipid profile and menstrual cycle with acne vulgaris. Clin Biochem. 2010;43(18):1415-1420.

34. Chang RJ, Katz SE. Diagnosis of polycystic ovary syndrome. Endocrinol Metab Clin North Am. 1999;28(2):397-408.

35. Goulden V, Clark SM, Cunliffe WJ. Post-adolescent acne: a review of clinical features. Br J Dermatol. 1997;136(1):66-70.

36. Housman E, Reynolds RV. Polycystic ovary syndrome: a review for dermatologists: Part I. Diagnosis and manifestations. J Am Acad Dermatol. 2014;71(5):847.

37. Lee WJ, Jung HD, Lee HJ et al. Influence of substance-P on cultured sebocytes. Arch Dermatol Res. 2008;300(6):311-317.

38. Makrantonaki E, Ganceviciene R, Zouboulis CC. An update on the role of the sebaceous gland in the pathogenesis of acne. Dermatoendocrinol. 2011;3(1):41-49.

39. Slominski AT, Zmijewski MA, Zbytek B, et al. Key role of CRF in the skin stress response system. Endocr Rev. 2013;34(6):827-884.

40. Huraseva AB. The search for ways to correct the syndrome of hyperandrogenism of nodules born with a large body mass. Obstetrics and gynecology. 2009;(5):68-71. 